\title{
Lipid Extraction by $\alpha$-Synuclein Generates Semi-Transmembrane Defects and Lipoprotein Nanoparticles
}

\author{
Jianjun Pan, ${ }^{* \dagger}{ }^{\dagger}$ Annalisa Dalzini, ${ }^{\ddagger}$ Nawal K. Khadka, ${ }^{\dagger}$ Chinta M. Aryal, ${ }^{\dagger}$ and Likai Song ${ }^{*}$, \\ ${ }^{\dagger}$ Department of Physics, University of South Florida, Tampa, Florida 33620, United States \\ ${ }^{\ddagger}$ National High Magnetic Field Laboratory, Florida State University, Tallahassee, Florida 32310, United States
}

\section{Supporting Information}

\begin{abstract}
Modulations of synaptic membranes play an essential role in the physiological and pathological functions of the presynaptic protein $\alpha$-synuclein $(\alpha$ Syn). Here we used solution atomic force microscopy (AFM) and electron paramagnetic resonance (EPR) spectroscopy to investigate membrane modulations caused by $\alpha$ Syn. We used several lipid bilayers to explore how different lipid species may regulate $\alpha \mathrm{Syn}-$ membrane interactions. We found that at a protein-to-lipid ratio of $\sim 1 / 9, \alpha$ Syn perturbed lipid bilayers by generating semi-transmembrane defects that only span one leaflet. In addition, $\alpha$ Syn coaggregates with lipid molecules to produce $\sim 10 \mathrm{~nm}$-sized lipoprotein nanoparticles. The obtained AFM data are consistent with the apolipoprotein characteristic of $\alpha$ Syn. The role of anionic lipids was elucidated by comparing results from zwitterionic and anionic lipid bilayers. Specifically, our AFM measurements showed that anionic bilayers had a larger tendency of forming bilayer defects; similarly, our EPR measurements revealed that anionic bilayers exhibited more substantial changes in lipid chain mobility and

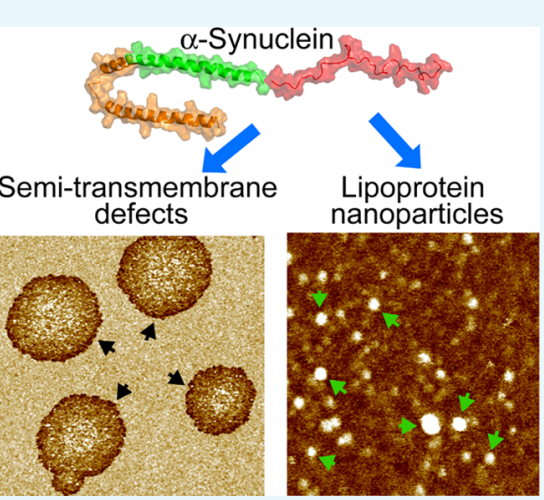
bilayer polarity. We also studied the effect of cholesterol. We found that cholesterol increased the capability of $\alpha$ Syn in inducing bilayer defects and altering lipid chain mobility and bilayer polarity. These data can be explained by an increase in the lipid headgroup-headgroup spacing and/or specific cholesterol- $\alpha$ Syn interactions. Interestingly, we found an inhibitory effect of the cone-shaped phosphatidylethanolamine lipids on $\alpha$ Syn-induced bilayer remodeling. We explained our data by considering interlipid hydrogen-bonding that can stabilize bilayer organization and suppress lipid extraction. Our results of lipid-dependent membrane modulations are likely relevant to $\alpha$ Syn functioning.
\end{abstract}

\section{INTRODUCTION}

The detrimental Parkinson's disease (PD) is featured by selective loss of dopaminergic neurons in the substantia nigra. ${ }^{1,2}$ One pathogenic hallmark of PD is the formation of filamentous aggregates known as Lewy bodies and Lewy neurites. ${ }^{3,4}$ Genome-wide association studies have shown that the rare form of familial PD is caused by missense mutations of a protein called $\alpha$-synuclein $(\alpha \operatorname{Syn}) .{ }^{5}$ Moreover, duplication ${ }^{6}$ and triplication ${ }^{7}$ of the SNCA gene encoding $\alpha$ Syn are sufficient to cause a highly penetrant form of PD. The causative role played by $\alpha \mathrm{Syn}$ in idiopathic $\mathrm{PD}$ is further substantiated by observations that intraneuronal deposits in $\mathrm{PD}$ brains are mainly composed of $\alpha \mathrm{Syn} .^{3}$

$\alpha$ Syn is a highly conserved cytosolic protein mainly localized at presynaptic nerve terminals. The 140-residue protein contains an $\mathrm{N}$-terminus harboring seven imperfect repeats of eleven-residue; the central region contains a hydrophobic domain referred to as NAC (non-A $\beta$ component); the Cterminus is negatively charged and largely unstructured. Similar to apolipoproteins, the eleven-residue repeats of $\alpha$ Syn enhance its membrane-binding affinity ${ }^{8}$ and may be responsible for aggregating with lipid molecules to form lipoprotein particles. ${ }^{9}$ Membrane association is also supported by secondary structure studies. $\alpha \operatorname{Syn}$ is largely unfolded in aqueous solution; peripheral membrane association converts the N-terminus of $\alpha$ Syn into an extended or broken $\alpha$-helix. ${ }^{10-12}$ Although not fully understood, both physiological and pathological functions of $\alpha$ Syn involve membrane interactions. ${ }^{13}$ For instance, overexpression of $\alpha$ Syn caused membrane disruption of mitochondria, ${ }^{14}$ Golgi apparatus, ${ }^{15}$ and endoplasmic reticulum. ${ }^{16}$

$\mathrm{PD}$ is featured by the accumulation of $\alpha$ Syn-dominated inclusion deposits in pathological brains. Accumulating evidence suggests that formation of fibrillar aggregates might be a cellular mechanism to cope with more toxic prefibrillar species. ${ }^{17-20}$ Indeed, high levels of $\alpha$ Syn are directly associated with PD pathology. ${ }^{6,7}$ Despite many strides being made in understanding the physiopathological functions of $\alpha \mathrm{Syn}^{21}$ the molecular mechanism of how $\alpha$ Syn gains cytotoxic properties and causes dopaminergic neuronal death remains unclear.

The amphipathic characteristic of $\alpha$ Syn confers its ability to interact with lipid membranes, ${ }^{22}$ yielding highly curved structures such as tubules, ${ }^{23-25}$ nanoparticles, ${ }^{9}$ and micelles. ${ }^{26}$ Membrane interactions of $\alpha$ Syn could lead to nerve cell

Received: June 26, 2018

Accepted: August 7, 2018

Published: August 21, 2018 
dysfunction by different mechanisms. One possibility is that specific lipids facilitate the generation of toxic $\alpha$ Syn aggregates (e.g., oligomers). A second possibility is based on the duplication and triplication of the SNCA gene. The resulting abnormal accumulation of $\alpha$ Syn might interfere with the normal membrane fission-fusion dynamics that are essential to synaptic vesicle cycling. A third possibility is that $\alpha \mathrm{Syn}$ participates in synaptic vesicle cycling by promoting membrane curvature remodeling; overexpression of $\alpha$ Syn could cause an imbalance between the vesicle exocytosis and endocytosis. Together, a better understanding of the mechanism of synaptic membrane interactions of $\alpha$ Syn will help elucidate both the physiological and pathological functions of $\alpha$ Syn.

In this work, we used high-resolution atomic force microscopy (AFM) and electron paramagnetic resonance (EPR) spectroscopy to investigate changes in lipid bilayer material properties caused by $\alpha$ Syn. Our AFM measurements can visually detect nanoscale changes in lipid membrane topographic structures, and our EPR measurements probe molecular-level lipid mobility and bilayer polarity. The obtained experimental data highlight the concept that $\alpha$ Syn can carry out its physiopathological functions by altering membrane properties. Many lipids play key roles in synaptic vesicle cycling. ${ }^{27}$ Lipid-specific interactions of $\alpha$ Syn might interfere with synaptic vesicle cycling. To elucidate different synaptic lipids that might be responsible for $\alpha \mathrm{Syn}-$ membrane interactions, we selectively studied lipid bilayers containing 1palmitoyl-2-oleoyl-sn-glycero-3-phosphatidylcholine (POPC), 1-palmitoyl-2-oleoyl-sn-glycero-3-phospho-(1'-racglycerol) (POPG), 1-palmitoyl-2-oleoyl-sn-glycero-3-phosphatidylethanolamine (POPE), and cholesterol (Chol) lipids. By determining the impacts of $\alpha$ Syn on physical properties of lipid bilayers containing different lipid species, our study provides useful insights into the role of various synaptic lipids in regulating $\alpha$ Syn-membrane interactions.

\section{RESULTS}

2.1. AFM Experiments on Planar Lipid Bilayers. 2.1.1. $P O P C / P O P G$ Bilayer. We used AFM imaging to visualize the effect of $\alpha \mathrm{Syn}$ on topographical structures of micasupported planar bilayers composed of POPC/POPG 4:1. (Unless noted otherwise, all ratios and percentages are molar based in this paper.) AFM height image of the control bilayer is shown in Figure 1A. The bilayer exhibited a homogeneous organization over the $10 \mu \mathrm{m} \times 10 \mu \mathrm{m}$ area. After confirming the quality of the intact bilayer, we used a syringe pump to inject a $1 \mu \mathrm{M} \alpha$ Syn solution into the AFM fluid cell. The protein incubation time was set to zero right after the injection. After incubation of $39 \mathrm{~min}$, sporadic defects with a circular shape and a length scale of $\sim 2 \mu \mathrm{m}$ were observed (Figure 1B). The image at $74 \mathrm{~min}$ revealed similar micron-scale defects, accompanied by a few smaller ones with a length scale of a few hundred nanometers. Similar micron-scale defects were observed after incubation of $139 \mathrm{~min}$. On the other hand, the in situ measurements revealed that the surface area fraction covered by the defects increased with the incubation time. This is quantified by image analysis showing that the defect area fraction is $0.14,0.20$, and 0.41 at the incubation times of 39 , 74, and $139 \mathrm{~min}$, respectively.

AFM probes lipid bilayer structures in both horizontal and vertical directions. The sub- $\AA$ sensitivity along bilayer normal enables us to determine the height profile of $\alpha$ Syn-induced defects (Figure 2). We found that the edge of the circularly
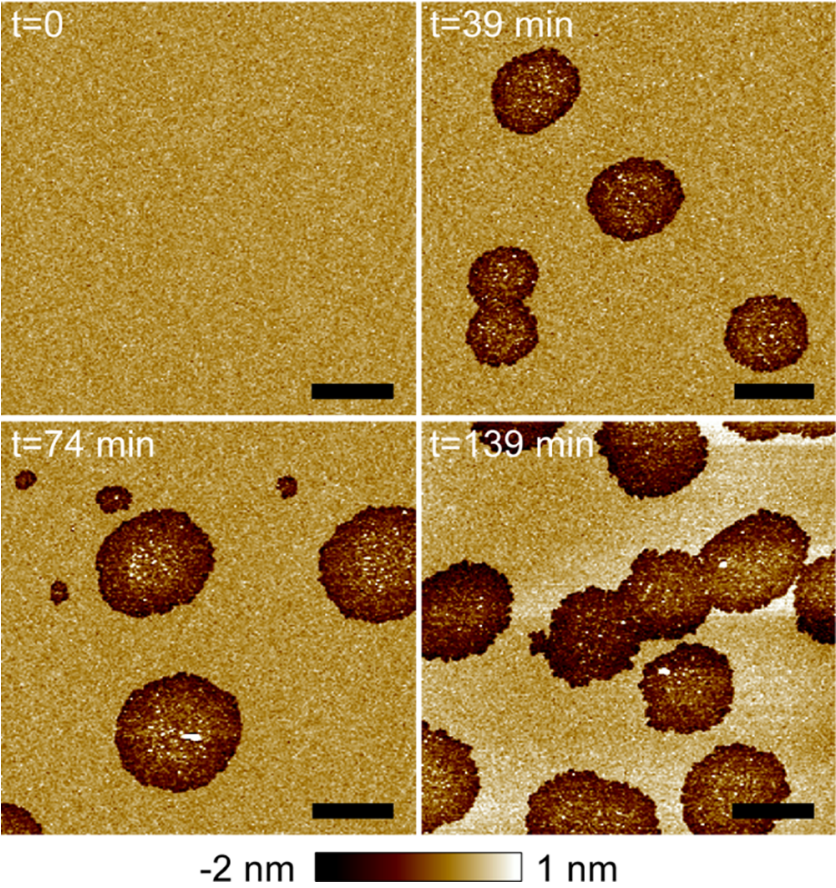

Figure 1. AFM images of a POPC/POPG 4:1 bilayer exposed to 1 $\mu \mathrm{M} \alpha \mathrm{Syn}$ as a function of the incubation time. Scale bars are $2 \mu \mathrm{m}$. Image height scale is indicated by the color bar at the bottom. The same color bar (with modified height scale if noted) is applied throughout the paper for AFM images. The area fractions of $\alpha$ Syninduced semi-transmembranedefectsare $0.14,0.20$, and 0.41 as theincubation time increases from 39 to $139 \mathrm{~min}$.
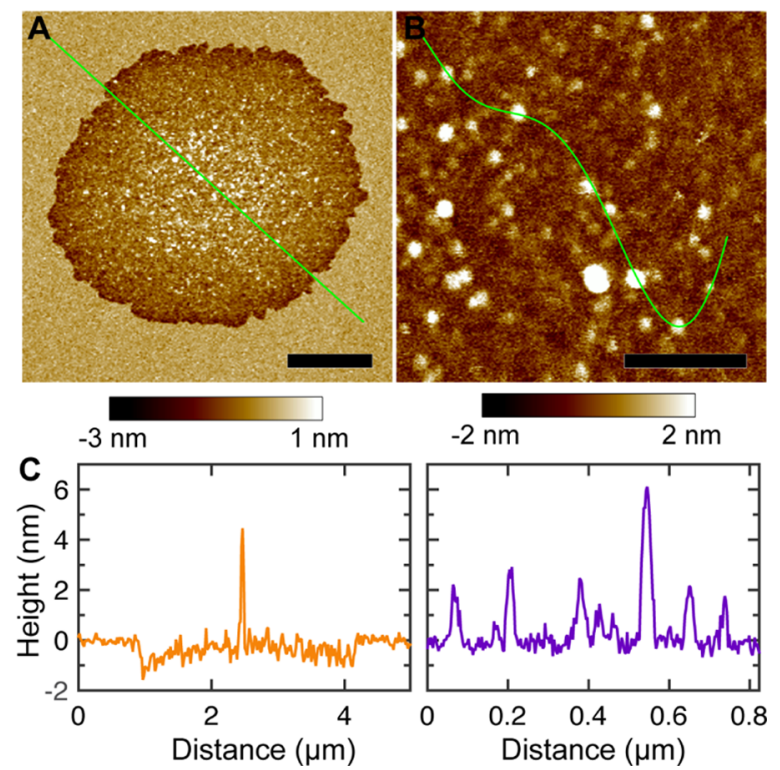

Figure 2. (A, B) High-resolution AFM images of a POPC/POPG 4:1 bilayer exposed to $1 \mu \mathrm{M} \alpha$ Syn. Scale bar is $1 \mu \mathrm{m}$ for (A) and $0.2 \mu \mathrm{m}$ for (B). (C) Height profiles along the solid green lines in (A) and (B).

shaped defects has a depth of $\sim 1-1.5 \mathrm{~nm}$ below the bilayer surface. Considering that the thickness of POPC and POPG bilayers is $\sim 3 \mathrm{~nm},{ }^{28,29}$ the observed defects only span one leaflet of the mica-supported bilayer. We also noticed that the interior of the defects contained many inhomogeneous features. To explore the detailed structure inside bilayer defects, we performed high-resolution AFM scans. Although 


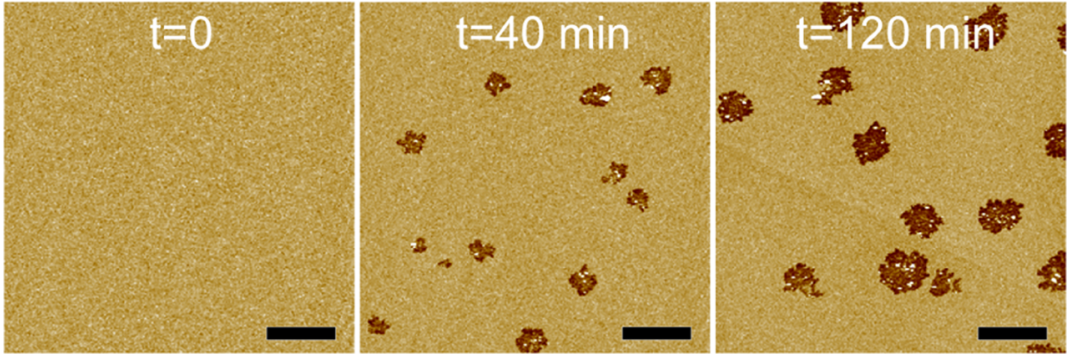

Figure 3. AFM images of a POPC bilayer exposed to $1 \mu \mathrm{M} \alpha \mathrm{Syn}$ as a function of the incubation time. Height scale is $4 \mathrm{~nm}$. Scale bars are $2 \mu \mathrm{m}$. The area fractions of $\alpha \mathrm{Syn}$-induced semi-transmembrane defects are 0.04 and 0.10 at the incubation times of 40 and 120 min, respectively.

not evident in micron-scale images, numerous nanoscale particles were clearly seen in the high-resolution image (Figure 2B). To estimate the height of the observed particles, we selected an arbitrary path crossing several particles (Figure 2B). Height profile along the path showed that the nanoscale particles have a height of $\sim 2-6 \mathrm{~nm}$ (Figure 2C).

We next performed image analysis to characterize particle sizes. A height threshold was used to distinguish particles from the background. (Pixels with heights larger than the threshold were considered as particles.) To account for pixels with occasional large heights (noise), a cutoff was used to discard particles smaller than $\sim 12 \mathrm{~nm}^{2}$. The resulting particles (i.e., bright regions in the binary image) are shown in Figure S1. Particle size was determined by the number of pixels (scaled by pixel size) occupied by each particle. Lastly, we converted the particle size to a particle radius by assuming that particles are circularly shaped. The probability distribution of the determined particle radius is shown in Figure S1. Gaussian curve fitting resulted in a most probable particle radius of 7 nm. Collectively, $\alpha$ Syn perturbed the POPC/POPG bilayer by forming semi-transmembrane defects; the interior of the defects was dispersed with many nanoscale particles, which have a height of $\sim 2-6 \mathrm{~nm}$ and an average radius of $7 \mathrm{~nm}$.

2.1.2. POPC Bilayer. We also used AFM imaging to study the effect of $\alpha$ Syn on zwitterionic POPC bilayers. Sporadic defects were observed after incubation with $1 \mu \mathrm{M} \alpha$ Syn for 40 and $120 \mathrm{~min}$ (Figure 3). Interestingly, the defects have serrated edges. Image analysis showed that the defect area fractions are 0.04 and 0.10 at the incubation times of 40 and $120 \mathrm{~min}$, respectively. These values are smaller compared to those of the POPC/POPG bilayer at similar incubation times. Figure 4 shows two high-resolution images encompassing one defect. Height profile indicated that POPC defects have a depth of $\sim 1.5 \mathrm{~nm}$ (Figure 4C). Circularly shaped particles were observed inside the defect. Height profile showed that the nanoscale particles have a height of $\sim 2-6 \mathrm{~nm}$. We selected a region inside the defect to estimate the particle size (Figure S2). Gaussian curve fitting showed that the most probable defect radius is $11 \mathrm{~nm}$.

2.1.3. POPC/Chol Bilayer. We used a POPC $+30 \%$ Chol bilayer to study the role of Chol in mediating $\alpha \mathrm{Syn}$-bilayer interactions. AFM images are shown in Figure 5. Circularly shaped defects were observed after exposing the bilayer to 1 $\mu \mathrm{M} \alpha$ Syn. The defect area fractions are 0.04 and 0.17 at the incubation times of 27 and $107 \mathrm{~min}$, respectively. Detailed defect structures were obtained by high-resolution scans focusing on one defect (Figure 6). Height profile showed that the defect depth is $\sim 2 \mathrm{~nm}$, slightly larger than the values for POPC/POPG and POPC bilayers. The interior of the defect also contained many particles. Height profile revealed
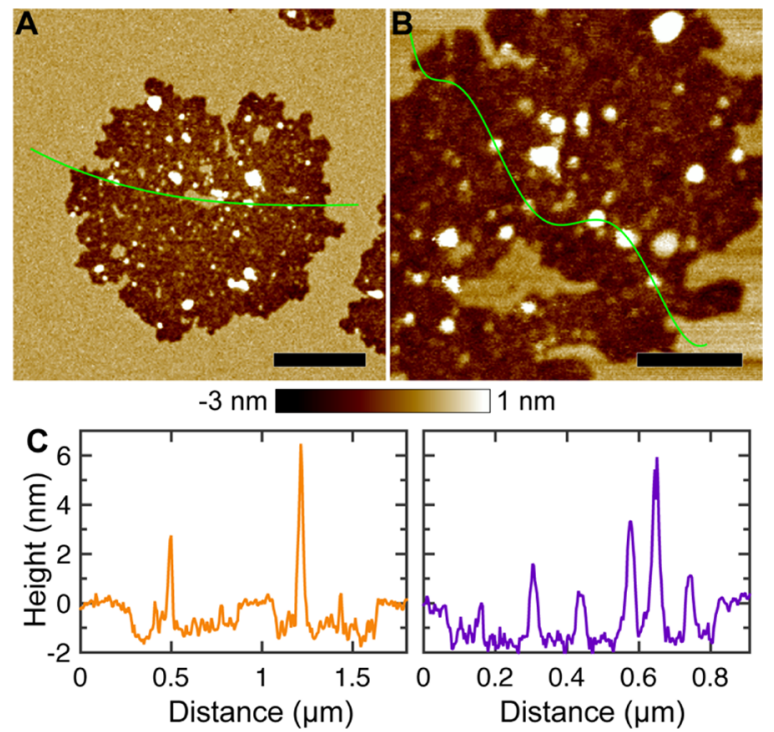

Figure 4. (A, B) High-resolution AFM images of a POPC bilayer treated with $1 \mu \mathrm{M} \alpha \mathrm{S} y$ n. The two images were obtained after incubation for $\sim 100 \mathrm{~min}$. Scale bar is $500 \mathrm{~nm}$ for (A) and $200 \mathrm{~nm}$ for (B). (C) Height profiles along the solid green lines shown in (A) and (B).

that the particles have a height of $\sim 2-6 \mathrm{~nm}$, the same range as for POPC/POPG and POPC bilayers. Image analysis showed that the particle radius distribution does not conform to a Gaussian distribution (Figure S3). Nevertheless, the average particle radius is about $10 \mathrm{~nm}$.

2.1.4. POPC/POPE Bilayer. We used POPC/POPE bilayers to study the role of $\mathrm{PE}$ lipids in modulating membrane perturbations caused by $\alpha$ Syn. Figure 7 shows the AFM images of a POPC $+15 \%$ POPE bilayer treated with $1 \mu \mathrm{M} \alpha \mathrm{Syn}$. Dispersed defects were observed as the incubation time increased from 22 to $105 \mathrm{~min}$. Longer incubation resulted in more defects. This is manifested by the defect area fractions, which are 0.02, 0.03, and 0.04 at the incubation times of 22, 58 , and $105 \mathrm{~min}$, respectively. Compared to the POPC bilayer, the defect area fraction is smaller for the POPC $+15 \%$ POPE bilayer at similar incubation times. Height profile showed that the defects in the POPC $+15 \%$ POPE bilayer have a depth of $\sim 1 \mathrm{~nm}$ (Figure 8). Nanoscale particles were also present in the interior of the defects. Height profile indicated that the particles have a height of $\sim 2-6 \mathrm{~nm}$. Particle size is estimated by image analysis shown in Figure S4. A most probable particle radius of $7 \mathrm{~nm}$ was obtained by Gaussian curve fitting. We also examined a POPC $+40 \%$ POPE bilayer. After incubation with $1 \mu \mathrm{M} \alpha \mathrm{Syn}$ for $106 \mathrm{~min}$, no discernable defects were observed 

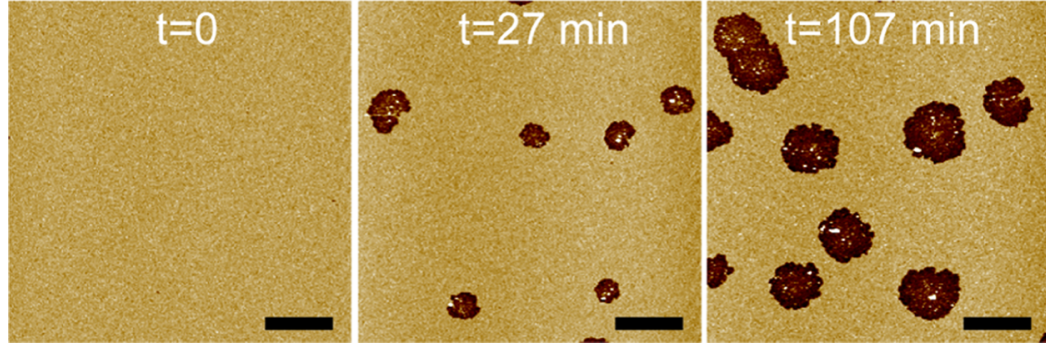

Figure 5. AFM images of a POPC $+30 \%$ Chol bilayer exposed to $1 \mu \mathrm{M} \alpha$ Syn as a function of the incubation time. Height scale is $4 \mathrm{~nm}$. Scale bars are $2 \mu \mathrm{m}$. The area fractions of $\alpha$ Syn-induced semi-transmembrane defects are 0.04 and 0.17 as the incubation time increases from 27 to 107 min.

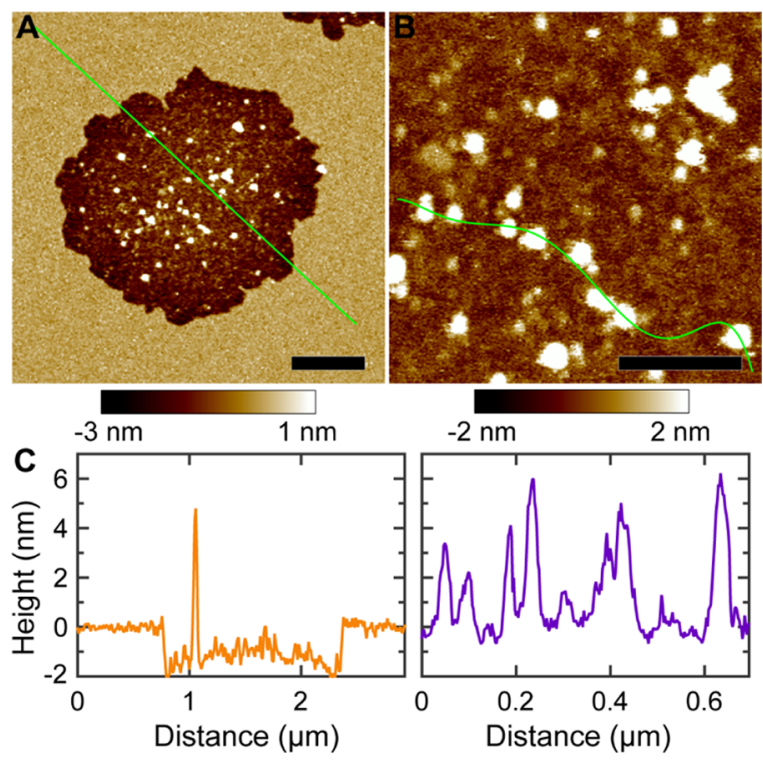

Figure 6. (A, B) High-resolution AFM images of a POPC $+30 \%$ Chol bilayer exposed to $1 \mu \mathrm{M} \alpha \mathrm{Syn}$. Scale bar is $500 \mathrm{~nm}$ for (A) and $200 \mathrm{~nm}$ for (B). (C) Height profiles along the solid green lines in (A) and $(\mathrm{B})$.
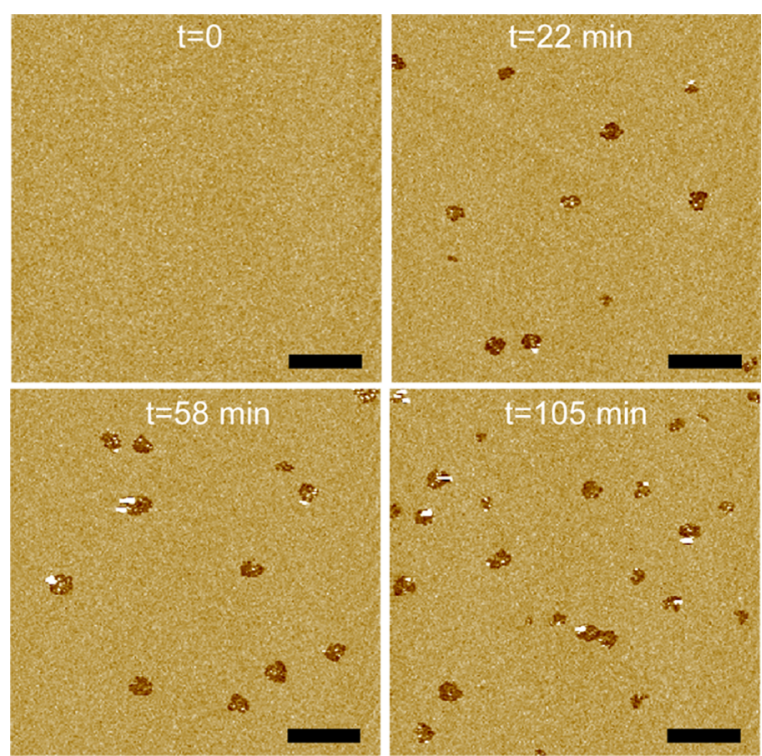

Figure 7. AFM images of a POPC $+15 \%$ POPE bilayer exposed to 1 $\mu \mathrm{M} \alpha \mathrm{Syn}$ as a function of the incubation time. Height scale is $4 \mathrm{~nm}$. Scale bars are $2 \mu \mathrm{m}$. The defect area fractions are 0.02, 0.03, and 0.04 as the incubation time increases from 22 to $105 \mathrm{~min}$.
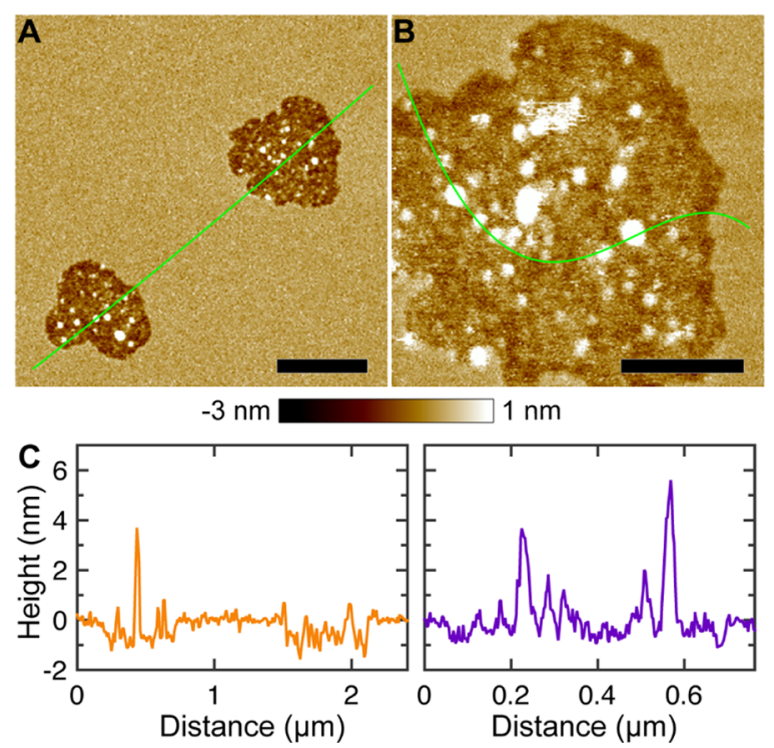

Figure 8. (A, B) High-resolution AFM images of a POPC $+15 \%$ POPE bilayer exposed to $1 \mu \mathrm{M} \alpha$ Syn. Scale bar is $500 \mathrm{~nm}$ for (A) and $200 \mathrm{~nm}$ for (B). (C) Height profiles along the solid green lines in (A) and (B).

(Figure S5). By comparing results from POPC/POPE bilayers with a varying content of POPE, we conclude that POPE suppressed the effect of $\alpha$ Syn in inducing semi-transmembrane defects.

2.2. EPR Spectroscopy Experiments on Lipid Vesicles. 2.2.1. Lipid Bilayer Fluidity. EPR spectra in the X-band ( 9.5 $\mathrm{GHz}$ ) were used to determine lipid bilayer fluidity (or mobility) changes upon $\alpha$ Syn binding. EPR spectral line shapes are influenced by molecular motion and therefore reflect lipid fluidity. ${ }^{30,31}$ In particular, an increase in the spectral peak-to-peak splitting and/or a broadening of the spectral line shape indicate slower molecular motion. To assess mobility changes induced by $\alpha$ Syn binding, we chose 5-doxyl stearic acid (5-SASL) as the spin probe because position 5 of the acyl chain has been demonstrated to be the most sensitive to mobility changes in several molecular models. ${ }^{32,33}$ We note that EPR spectroscopy mainly reports an averaged change of the microenvironment near the spin probe. Here, lipid mobility changes were defined using the motional parameter $2 A_{/ /}$, the peak-to-peak splitting of the spectrum, upon $\alpha$ Syn binding to lipid bilayers with different compositions. The protein-to-lipid $(\mathrm{P} / \mathrm{L})$ ratios investigated range from $1 / 200$ to $1 / 50$. As illustrated in Figures 9 and S6, the $2 A$ // values were increased for negatively charged POPC/POPG 4:1 bilayers upon $\alpha$ Syn binding, indicating a decreased lipid mobility. A comparable 


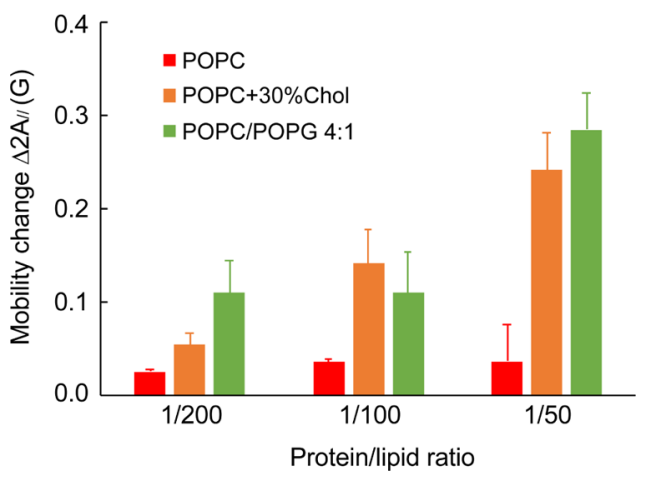

Figure 9. Lipid mobility changes for POPC (red), POPC $+30 \%$ Chol (orange), and POPC/POPG 4:1 (green) bilayers in the presence of $\alpha$ Syn at three $\mathrm{P} / \mathrm{L}$ ratios. The unit for the mobility change is Gauss. Greater $2 A_{/ /}$values reflect lower lipid mobility and membrane fluidity.

decrease in lipid mobility was observed for POPC bilayers with $30 \%$ Chol. Conversely, minimal changes were observed for zwitterionic POPC bilayers.

2.2.2. Lipid Bilayer Polarity. The effect of $\alpha$ Syn on lipid bilayer polarity was assessed by EPR power saturation and solvent accessibility experiments. ${ }^{34}$ Because a polarity gradient is present across lipid bilayers, nonpolar molecules are more accessible to lipid acyl chains, while polar agents accumulate in the solvent and around the polar or charged headgroups of phospholipids. The equilibrium can be perturbed by molecules that modulate the bilayer structure and organization. EPR power saturation experiments are suitable to evaluate the polarity of lipid bilayers and how the polarity is perturbed by macromolecules. To achieve this information, we exploited the saturation of the EPR signal of a nitroxide placed at a specific position (5-SASL) of the acyl chain with increasing microwave power, which can be quantified using the saturation parameter $P_{1 / 2} . P_{1 / 2}$ values in the presence of the nonpolar reagent $\mathrm{O}_{2}$ and the polar reagent nickel(II) ethylenediaminediacetate (NiEDDA) can be used to calculate a depth parameter $\Phi$, which reflects the polarity at a given position of the lipid bilayer. Figure 10 reports the $\Phi$ values for POPC, POPC/POPG 4:1, and POPC $+30 \%$ Chol bilayers with trace amounts of 5-SASL in the absence and presence of $\alpha$ Syn at $\mathrm{P} / \mathrm{L}=1 / 100$. To be noted, the $\Phi$ values for the bilayers without $\alpha$ Syn are comparable between POPC and POPC/POPG 4:1, while the $\Phi$ value is larger for POPC $+30 \%$ Chol. The larger $\Phi$ value in the presence of $\mathrm{Chol}$ is caused by an enhanced lipid chain order and a reduced solvent penetration. The addition of $\alpha$ Syn caused no significant changes in the $\Phi$ value for POPC bilayers, confirming the weak perturbations observed in the mobility experiment (Figure 9). On the contrary, there is a considerable decrease in the $\Phi$ value for POPC/POPG bilayers upon $\alpha$ Syn binding (35\%) and an even larger decrease for POPC $+30 \%$ Chol bilayers (43\%). These data indicate that $\alpha$ Syn interacts strongly with POPC/POPG 4:1 and POPC + $30 \%$ Chol bilayers, causing more polar solvent penetration.

\section{DISCUSSION}

3.1. Mechanism of Bilayer Perturbation by $\alpha$ Syn. We used solution AFM to explore structural remodeling of several
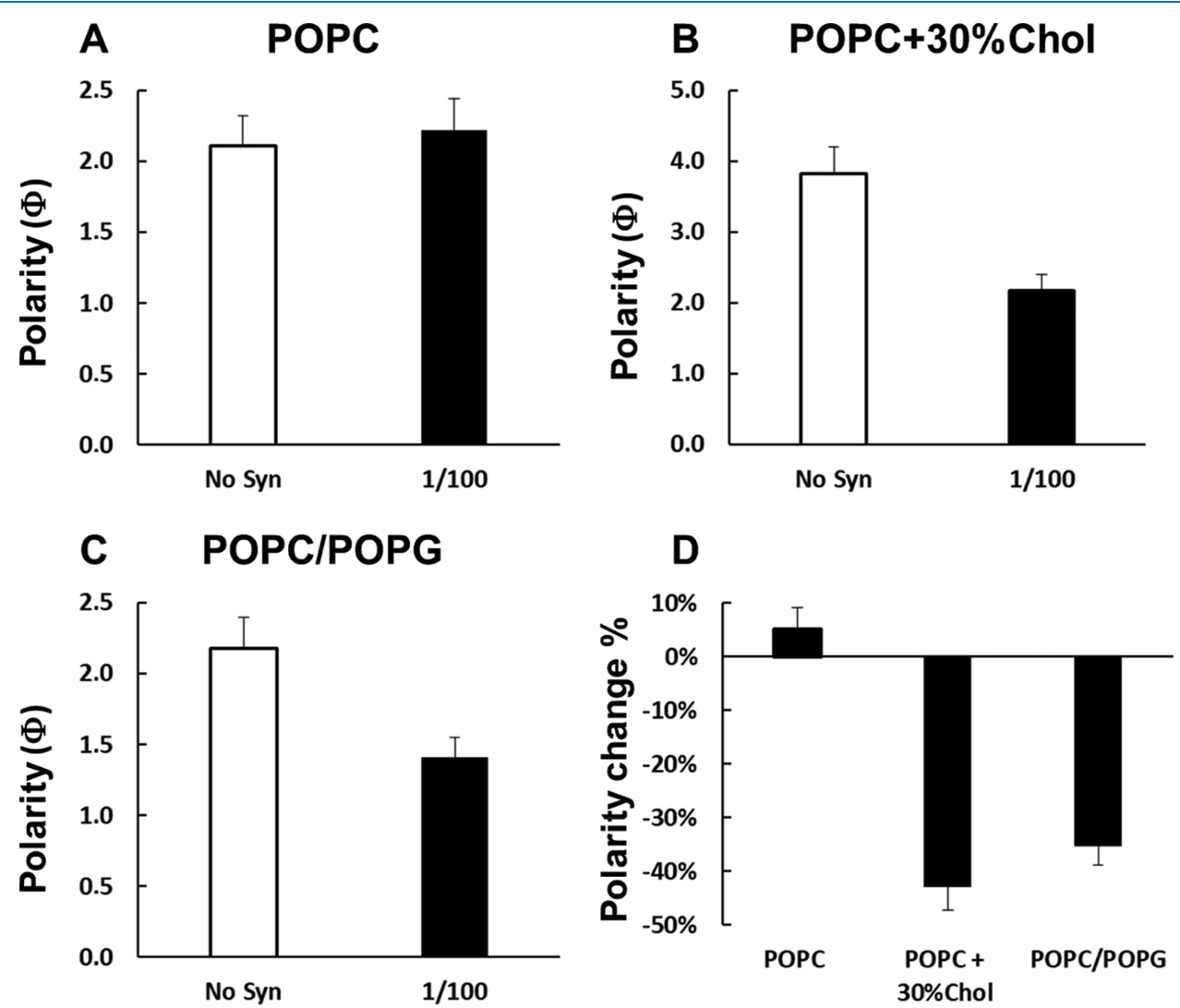

Figure 10. Membrane polarity changes upon the binding of $\alpha$ Syn for POPC (A), POPC $+30 \%$ Chol (B), and POPC/POPG 4:1 (C) bilayers at P/ $\mathrm{L}=1 / 100$. (D) Comparison of the percentage of the $\Phi$ value changes of the three bilayers in the presence of $\alpha$ Syn. Greater $\Phi$ values indicate lower polarity. 
lipid bilayers induced by $\alpha$ Syn. A common theme is the observation of micron-scale semi-transmembrane defects after treating planar bilayers with $1 \mu \mathrm{M} \alpha \mathrm{Syn}-$ aggregation of $\alpha$ Syn occurs at much higher concentrations even in the presence of anionic lipids. ${ }^{35}$ Pore-like structures induced by $\alpha$ Syn have been reported earlier. ${ }^{36,37}$ Our AFM measurements provide the first evidence that $\alpha$ Syn only causes semi-transmembrane defects, not membrane-spanning pores. The formation of semitransmembrane defects is based on the measurement of defect depth, which is $\sim 1-2 \mathrm{~nm}$. Note that we have used AFM imaging and obtained transmembrane defects with a depth of $\sim 4 \mathrm{~nm}$ when exposing lipid bilayers to an amphipathic Prion peptide. ${ }^{38}$ Generation of semi-transmembrane defects requires removal of lipid molecules from the top leaflet of the planar bilayer. Therefore, our AFM data support the mechanism of lipid extraction by $\alpha$ Syn. Many amphipathic peptides have a similar ability to extract lipid molecules from bilayer assemblies, ${ }^{39,40}$ a mechanism known as the detergent effect. After binding to lipid membranes, $\alpha$ Syn transits from a disordered structure to a helix-enriched conformation. ${ }^{8}$ Depending on the membrane curvature, $\alpha$ Syn can form an extended helix ${ }^{12}$ or two broken helices. ${ }^{10}$ In either case, $\alpha$ Syn exhibits an amphipathic characteristic where hydrophobic residues are segregated on one face, and polar and charged residues are enriched on the opposite face. The amphipathic characteristic of $\alpha \mathrm{Syn}$ is likely to be responsible for the lipid extraction effect observed in our study. The lipid extraction effect of $\alpha$ Syn is also supported by other studies. For example, $\alpha$ Syn caused fragmentation of giant unilamellar vesicles; ${ }^{41}$ $\alpha$ Syn can act as a vesicle fusion promoter. ${ }^{42}$ Both bilayer fragmentation and vesicle fusion require lipid extraction from the parent bilayer.

Because we only observe defects formed in one leaflet, it seems that $\alpha$ Syn does not have the ability to translocate from one leaflet to the other. This result is in line with studies showing that $\alpha$ Syn only inserted into the proximal leaflet where the protein was administrated. ${ }^{41}$ Insertion of $\alpha \mathrm{Syn}$ (at low concentrations before the formation of bilayer defects) into one leaflet will cause an area expansion of the target leaflet, whereas the area of the other leaflet will remain unchanged. ${ }^{43}$ The asymmetric area expansion across the coupled leaflets might cause bilayer bulging and tubulation. $^{23,25,26}$

Within each semi-transmembrane defect, the removal of the top leaflet by $\alpha$ Syn will cause lipid acyl chains of the bottom leaflet being exposed to water. Such an arrangement is energetically unfavorable. We can speculate several mechanisms to counteract the unfavorable hydrophobic effect of exposed lipid acyl chains. Our AFM imaging showed that the number of particles in the interior of the defect is fairly large. These particles can cover part of the exposed lipid acyl chains. $\alpha$ Syn monomers that did not participate in particle formation can contribute to shielding the exposed lipid acyl chains by residing on the surface of the bottom leaflet. The lipids extracted from the top leaflet may interdigitate with the bottom leaflet to form a compacted pseudo-monolayer. Lastly, lipid molecules at the bottom leaflet may have a slow desorption rate from the mica surface. This will result in a temporarily trapped pseudo-monolayer.

In addition to semi-transmembrane defects, we report $\sim 10$ nm-sized particles formed in the interior of the defects. Two explanations can account for the obtained nanoparticles. One is the formation of $\alpha$ Syn oligomers after binding to lipid bilayers. However, the size of the nanoparticles is larger than those of soluble oligomers prepared from monomeric $\alpha$ Syn. $^{44,45}$ Moreover, protein aggregation of $\alpha$ Syn takes a longer time (e.g., a few days) than the duration of our AFM experiment. $^{46}$ The most likely cause for the observed nanoparticles is that $\alpha$ Syn coaggregates with lipid molecules to form the $\sim 10 \mathrm{~nm}$-sized nanoparticles. The possibility of forming lipoprotein nanoparticles is corroborated by the apolipoprotein motif of $\alpha$ Syn. Moreover, 7-10 nm-sized lipoprotein nanoparticles were reported when incubating $\alpha$ Syn with lipid vesicles. ${ }^{9}$

The lipid extraction by $1 \mu \mathrm{M} \alpha$ Syn likely represents a later event following protein binding. It is conceivable that when the concentration of $\alpha$ Syn becomes smaller, the probability of developing semi-transmembrane defects will be diminished. This is confirmed by our AFM experiments when exposing POPC/POPG 4:1 bilayers to $\alpha$ Syn solutions containing lower protein concentrations. Specifically, we found no defects at 0.2 $\mu \mathrm{M} \alpha \mathrm{Syn}$ and a few defects at $0.5 \mu \mathrm{M} \alpha \mathrm{Syn}$ (data not shown). The observed trend is consistent with the theory that there is a minimum number of lipid molecules that are required to completely bind one $\alpha$ Syn molecule. ${ }^{47}$ Depending on the lipid bilayer composition, the minimum number was estimated to be $\sim 85-2000$ lipids per $\alpha$ Syn. ${ }^{47}$ Bilayer destabilization can occur when the number of lipids is smaller than the required minimum lipid number. We estimated the $\mathrm{P} / \mathrm{L}$ ratio (and the number of lipids per $\alpha$ Syn) used in our AFM experiments. The fluid cell diameter is $\sim 8 \mathrm{~mm}$. Assuming an area per lipid of $0.62 \mathrm{~nm}^{2}$, the total number of lipids in one mica-supported bilayer is $1.62 \times 10^{14}$. Assuming a fluid cell volume of $30 \mu \mathrm{L}$, the corresponding $\mathrm{P} / \mathrm{L}$ ratios are $1 / 9,1 / 18$, and $1 / 45$ at $1,0.5$, and $0.2 \mu \mathrm{M} \alpha \mathrm{Syn}$, respectively. These values indicate that at 1 $\mu \mathrm{M} \alpha \mathrm{Syn}$, the number of lipids per $\alpha \mathrm{Syn}$ is smaller than the lower boundary of the required minimum lipid number (i.e., 85 ). Due to the insufficiency of lipid molecules to completely bind all $\alpha$ Syn molecules, bilayer disruption (e.g., lipid extraction) can be elicited by excess $\alpha$ Syn. Therefore, our AFM data agree with the theory of the minimum lipid number per bound $\alpha$ Syn. ${ }^{47}$

Our EPR experiments were performed at low $\mathrm{P} / \mathrm{L}$ ratios where the probability of lipid extraction by $\alpha$ Syn is small. Therefore, the obtained EPR data mostly reflect bilayer structural changes before the formation of semi-transmembrane defects. Another aspect is that spin probes at the inner and outer leaflets of lipid vesicles are likely to be affected differently by $\alpha$ Syn because the protein was added to the outside of the vesicles. Consequently, the obtained EPR data reflect an averaged effect of $\alpha$ Syn on lipid bilayer properties. For all the three bilayers studied, we observed an increase in the spectral peak-to-peak splitting, although the increase was much larger for POPC/POPG and POPC/Chol than for POPC. An increase in the peak-to-peak splitting indicates that $\alpha$ Syn reduced lipid mobility and bilayer fluidity. A similar result was reported by an earlier EPR spectroscopy study, which showed that $\alpha$ Syn restricted the segmental rotational mobility and increased the chain order of an anionic fluid bilayer. ${ }^{48}$ A reduction in bilayer fluidity was also reported by measuring changes in the general polarization of POPG bilayers. ${ }^{49}$ Computational simulations have predicted somewhat conflicting results. Both chain ordering ${ }^{50}$ and disordering $^{43}$ were reported by the same group. Overall, $\alpha$ Syn can modulate structural and dynamical properties of lipid bilayers. Depending on the mode of bilayer interactions (e.g., bilayer 
penetration depth), the resulting changes in lipid bilayer properties can vary. For example, a shallow binding to the headgroup region is likely to have less impact on bilayer chain organization than a deep penetration into the hydrocarbon core.

3.2. Effect of Anionic Lipids. Anionic lipids are important constituents of presynaptic nerve terminals, where cytosolic $\alpha$ Syn is located. Our AFM data of POPC and POPC/POPG bilayers showed that $\alpha \mathrm{Syn}$ caused a larger degree of bilayer perturbation when anionic lipid POPG was present. This is reflected in the difference of the defect area fraction. The edge of the defects also seems different. A smoother edge in the presence of POPG may represent a larger degree of the cooperativity of $\alpha \mathrm{Syn}$ molecules in generating bilayer defects. Despite these differences, the nanoparticles produced by $\alpha$ Syn have a similar size scale (i.e., $\sim 10 \mathrm{~nm}$ radius) without or with $20 \%$ POPG. A larger degree of bilayer perturbation in the presence of POPG is also observed in our EPR experiments. In particular, spectral line-shape measurements revealed that $\alpha$ Syn caused a larger decrease in lipid mobility for the POPC/POPG than for the POPC bilayer. Similarly, EPR power saturation measurements showed a more significant increase in bilayer polarity when POPG was present. Our observation of a larger effect of $\alpha$ Syn on lipid bilayers containing POPG is consistent with the long-known role of anionic lipids in regulating $\alpha$ Syn-membrane interactions. $\alpha$ Syn adopts a helical conformation after binding to lipid membranes. ${ }^{8}$ Helical wheel analysis showed that many basic residues are located at the interface between the polar and nonpolar faces. ${ }^{8,11}$ The basic characteristic of $\alpha \mathrm{Syn}$ is the primary cause for its marked preference of binding to membranes containing anionic lipids. ${ }^{8,47}$ The strong binding of $\alpha \mathrm{Syn}$ to anionic lipids may modulate the kinetics of $\alpha \mathrm{Syn}$ aggregation. ${ }^{35}$ Interestingly, not only the number of charges but also the position of the basic residues was found to affect membrane association of $\alpha$ Syn. ${ }^{51}$ Introduction of basic residues on the hydrophobic face of $\alpha$ Syn hindered its conformational change and diminished its membrane binding. ${ }^{51}$

3.3. Effect of Chol. Synaptic vesicles have a high Chol content. $^{52}$ Although the function of vesicular Chol remains to be defined, Chol likely participates in synaptic vesicle cycling. ${ }^{53}$ At presynaptic plasma membranes, Chol was found to be required for vesicle biogenesis. ${ }^{54} \mathrm{Chol}$ can affect protein functioning by modulating membrane material properties. ${ }^{55-57}$ In addition, Chol can act as a ligand by directly binding to proteins. Many studies have linked Chol to chronic neurodegenerative disorders. ${ }^{58}$

By monitoring monolayer pressure, Fantini et al. found that $\alpha$ Syn had a high affinity for Chol. ${ }^{59}$ The authors also identified a segment of $\alpha \operatorname{Syn}$ (residues 67-78) called "titled peptide" that is hydrophobic and responsible for Chol binding. Molecular simulation revealed that the binding was facilitated by structural complementary between the rough $\beta$-face of Chol and the hydrophobic pocket of the titled peptide. ${ }^{59}$ In addition, the $\mathrm{N}$-terminus of $\alpha$ Syn (residues 34-45) contains a Chol recognition consensus motif. The consensus domain was attributed as the second site for Chol binding, albeit with a weaker affinity. ${ }^{59}$ Chol binding may account for Chol efflux caused by exogenous $\alpha \mathrm{Syn} .{ }^{60} \mathrm{Chol}$ binding may also increase the local concentration of $\alpha$ Syn on membrane surfaces; the augmented protein-protein interactions may then lead to Chol-stimulated $\alpha$ Syn aggregation. ${ }^{61}$
In addition to direct binding, Chol may regulate $\alpha \mathrm{Syn}-$ membrane interactions by expanding the lipid headgroupheadgroup spacing; the increased vacancy in the headgroup region may then better accommodate $\alpha$ Syn binding and insertion. An analogy to Chol-induced expansion of the lipid headgroup-headgroup spacing can be found when studying $\alpha$ Syn binding to lipid unilamellar vesicles with different radii. $\alpha$ Syn has a stronger binding affinity to vesicles with smaller radii. ${ }^{8,62}$ The effect of vesicle curvature (radius) on $\alpha$ Syn binding can be understood by considering the intrinsic defects (e.g., vacancy) at the bilayer-water interface. ${ }^{63}$ Vesicles with larger curvature (i.e., smaller radius) have more intrinsic defects to facilitate $\alpha$ Syn binding. ${ }^{64}$

The effect of Chol is revealed by our AFM and EPR measurements on POPC and POPC $+30 \%$ Chol bilayers. Our AFM experiment showed that $\alpha$ Syn induced semi-transmembrane defects in both bilayers; the defect area fraction was larger for POPC/Chol than for POPC bilayers. In parallel, our EPR experiments showed that $\alpha$ Syn caused larger changes in lipid mobility and bilayer polarity when Chol was present. The enhanced bilayer perturbation in the presence of Chol can be caused by a higher bilayer binding affinity due to Chol- $\alpha$ Syn recognition. ${ }^{59}$ Alternatively, Chol expands lipid headgroup-headgroup spacing, ${ }^{65}$ allowing more $\alpha$ Syn molecules to insert into the headgroup region. The lipid mobility and bilayer polarity data from our EPR measurements are closely related to lipid chain organization. Because $\alpha$ Syn caused significant changes in lipid mobility and bilayer polarity of POPC/POPG and POPC/Chol, it is likely that (part of) $\alpha$ Syn penetrated deeper beyond the lipid headgroup region. This view is supported by reports from neutron reflection measurements. ${ }^{6,67}$

3.4. Effect of PE Lipids. PE lipids are the second most abundant phospholipids in mammalian cells. It accounts for $20-50 \mathrm{~mol} \%$ of the total phospholipids; a content of $\sim 45 \mathrm{~mol}$ $\%$ is observed in the brain. ${ }^{68}$ One unique characteristic of PE lipids is that the polar headgroup has a smaller in-plane area than that of the hydrophobic acyl chains. The difference in the in-plane areas renders PE lipids a conical shape. Concomitantly, lipid assemblies (e.g., monolayer and bilayer) composed of PE lipids have a negative spontaneous curvature. ${ }^{69}$ In a bilayer assembly with large curvatures as exemplified by the synaptic nerve terminals, PE lipids are preferentially packed at the inner leaflet of the convex membrane. The second important feature of PE lipids resides in the amine group, which is a good hydrogen-bond donor. Interlipid hydrogen-bonding between the amine moiety of PE lipids and the phosphate oxygen (and carbonyl oxygen) of neighboring lipids contributes to an enhanced bilayer stability. ${ }^{70,71}$ A structural manifestation of the hydrogenbonding is that PE lipids have smaller areas per lipid than those of PC counterparts. ${ }^{72}$ Hydrogen-bonding renders PEcontaining membranes more resilient to perturbations imparted by external agents. For example, PE lipids are less susceptible to ethanol-facilitated lipid desorption. ${ }^{33}$ Our recent study of an amphipathic peptide called M2AH derived from the influenza M2 protein also showed that POPE inhibited the activity of $\mathrm{M} 2 \mathrm{AH}$ in modulating lipid bilayer properties. ${ }^{74}$

An earlier study using thin-layer chromatography showed that $\alpha$ Syn bound to brain PE lipids; the authors also found that PE lipids in conjugation with anionic lipids, but not PE lipids alone, increased the helical content of $\alpha$ Syn. ${ }^{36}$ By monitoring birefringence and protein/lipid mass ratio, Ouberai et al. 
reported that cone-shaped PE lipids enhanced $\alpha$ Syn binding. ${ }^{75}$ Moreover, the binding affinity of $\alpha$ Syn to planar bilayers was larger than to curved vesicles. The authors attributed their findings to PE-induced packing defects. ${ }^{75}$

Here we used POPC/POPE bilayers to investigate the effect of PE lipids in response to $\alpha$ Syn perturbation. The experiment was performed at room temperature $\left(\sim 23{ }^{\circ} \mathrm{C}\right)$. Differential scanning calorimetry (DSC) measurements have been performed by Cannon et al. on POPC/POPE mixtures with the content of POPE ranging from 61 to $100 \% .{ }^{76}$ DSC peaks were located at $\sim 18$ and $22.4{ }^{\circ} \mathrm{C}$ for the POPE contents of 61 and $85 \%$, respectively. The highest content of POPE in our study is $40 \%$. Based on the relationship between the DSC peak position and the POPE content reported by Cannon et al., it is reasonable to deduce that the phase transition temperature for POPC $+40 \%$ POPE is lower than $18{ }^{\circ} \mathrm{C}$. Therefore, the gel-tofluid phase transition temperature for the POPC $+40 \%$ POPE bilayer is more than $5{ }^{\circ} \mathrm{C}$ below room temperature. Our AFM data suggested that POPE suppressed the capability of $\alpha$ Syn in inducing semi-transmembrane defects. This is reflected in the inverse relationship between the defect area fraction and the POPE content. Notably, no defects were observed at $40 \%$ POPE. Lipid extraction occurs during the formation of semitransmembrane defects. The interlipid hydrogen-bonding between PE and PE (or PC) lipids creates a network that can act against lipid extraction. Our view of suppressed lipid extraction by $\alpha$ Syn due to interlipid hydrogen-bonding is substantiated by the finding that PE lipids negated lipid extraction by ethanol. ${ }^{73}$ Along the same line, the antimicrobial peptide LL-37 was found to be inactive against PE monolayers. ${ }^{77}$ Our observation of the inhibitory effect of PE lipids on $\alpha$ Syn activity is in stark contrast to the report showing that PE lipids enhanced $\alpha$ Syn binding. ${ }^{75}$ One plausible explanation is that anionic lipids were used together with PE lipids in the binding assay. ${ }^{75}$ Lipid-lipid electrostatic interactions coexist with (and may disrupt) interlipid hydrogen-bonding. Therefore, the binding assay data cannot be solely accounted for by considering the effect of PE lipids. Indeed, a mixture of PE and anionic lipids was found to increase the helical content of $\alpha$ Syn, whereas PE lipids alone exerted no effect. ${ }^{36}$ A second possible explanation is that formation of semi-transmembrane defects observed in our study represents a later stage following $\alpha$ Syn binding. A larger binding affinity toward PE bilayers does not necessarily result in more bilayer defects because the binding and lipid extraction can be two isolated events.

Membrane curvature remodeling is a highly regulated process during synaptic vesicle trafficking. Due to the abundance of PE lipids at the inner leaflet of synaptic plasma membranes and the reduced membrane interactions between $\alpha$ Syn and PE-containing membranes, premature membrane remodeling caused by $\alpha$ Syn could be inhibited during synaptic vesicle cycling. Alternatively, displacement of PE lipids from synaptic active zones ${ }^{78}$ may promote $\alpha$ Syn-induced membrane reorganization. As the brain ages, the $\mathrm{PE}$ content in neuronal membranes can decline. In particular, a significantly lower PE content was reported in PD patients than in control subjects. ${ }^{79}$ PE deficiency may lead to impaired $\alpha$ Syn-membrane interactions, thus causing $\alpha$ Syn-associated abnormality. ${ }^{80}$

\section{CONCLUSIONS}

In this paper, we used high-resolution AFM and EPR spectroscopy to investigate membrane modulations caused by the cytosolic protein $\alpha$ Syn. In particular, we used lipid bilayers with several lipid compositions to explore different lipid species in regulating $\alpha$ Syn-membrane interactions. We found that at high $\mathrm{P} / \mathrm{L}$ ratios, $\alpha$ Syn perturbed lipid bilayers by forming micron-scale semi-transmembrane defects and $\sim 10$ nm-sized lipoprotein nanoparticles. The obtained results are consistent with the amphipathic characteristic and the apolipoprotein motif of $\alpha$ Syn. By comparing results from POPC and POPC/POPG bilayers, we confirm the role of anionic lipids in enhancing bilayer perturbations caused by $\alpha$ Syn. We found that Chol increased the capability of $\alpha$ Syn in inducing semi-transmembrane defects, decreasing lipid mobility, and increasing bilayer polarity. The results can be explained by an increase in the lipid headgroup-headgroup spacing or specific Chol- $\alpha$ Syn interactions. We found an inhibitory effect of the cone-shaped POPE lipids on $\alpha$ Syninduced membrane remodeling. We explained our data in the context of interlipid hydrogen-bonding that can stabilize bilayer organization and suppress lipid extraction. Lipiddependent selective membrane interactions are likely important for $\alpha$ Syn functioning. This is exemplified by PD-associated genetic mutants of $\alpha \mathrm{Syn}$ (A30P and A53T), which have weaker membrane binding affinities compared to the wild type. $^{81}$

\section{MATERIALS AND METHODS}

Lipids including POPC, POPG, POPE, and Chol were purchased from Avanti Polar Lipids (Alabaster, AL). 5-SASL was purchased from Sigma-Aldrich (St. Louis, MO). Human recombinant $\alpha$ Syn was purchased from Alexo-Tech $\mathrm{AB}$ (Sweden). Protein purity $>95 \%$ was verified by the vendor (high-performance liquid chromatography and sodium dodecyl sulfate-polyacrylamide gel electrophoresis). Fresh protein stock solutions were prepared by dissolving lyophilized protein powder in $10 \mathrm{mM} \mathrm{N}$-(2-hydroxyethyl)piperazine- $N^{\prime}$-ethanesulfonic acid (HEPES) pH 7.4. Centrifugation was used to remove preexisting fibrillar aggregates.

5.1. Atomic Force Microscopy (AFM) Imaging. Lipid mixtures were prepared by mixing appropriate ratios of lipid stock solutions in glass test tubes. Organic solvents (chloroform or chloroform/methanol) were removed by a gentle stream of argon gas, followed by vacuum pumping for $\sim 2 \mathrm{~h}$. Lipid dry films were hydrated in $10 \mathrm{mM}$ HEPES $\mathrm{pH}$ 7.4. Small unilamellar vesicles (SUVs) were produced by ultrasonication using a Sonic Dismembrator and a $3 \mathrm{~mm}$ microprobe. The obtained SUVs were centrifuged briefly before planar bilayer preparation. A Multimode 8 AFM (Bruker, Santa Barbara, CA) and a Nanoscope $\mathrm{V}$ controller were used for solution AFM imaging (room temperature). The experimental procedure has been reported elsewhere. ${ }^{38,82,83}$ Mica-supported planar bilayers were prepared by injecting lipid SUVs into a fluid cell where a freshly cleaved mica film acted as a solid substrate. SUVs in the vicinity of the mica film were attracted onto the mica surface, followed by vesicle rupture. ${ }^{84,85}$ Each ruptured vesicle will form a bilayer patch supported by the mica film. Because there were many vesicle rupture events taking place on the mica surface within a short period of time, a complete bilayer was formed by bilayer patch fusion within a few minutes. Excess SUVs that did not participate in bilayer formation were flushed out of the fluid cell by injecting the HEPES buffer. During the bilayer preparation process, the AFM tip was positioned at $\sim 50 \mu \mathrm{m}$ above the mica surface. The peak-force quantitative nanomechanics (QNM) mode was used for bilayer scanning with 
the peak force set to $\sim 300 \mathrm{pN}$. Square images were acquired using a silicon nitride probe DNP-S10 with a scan rate of $0.5-$ $1 \mathrm{~Hz}$. To eliminate potential artifacts induced by repetitive scanning, we manually moved the AFM tip to a different region after acquiring one or a few images at one location. The obtained topographic (height) images were leveled by subtracting a polynomial background. Image analysis was performed using in-house-developed Matlab scripts.

5.2. Electron Paramagnetic Resonance (EPR) Spectroscopy. 5.2.1. Vesicle Preparation. Unilamellar liposomes were prepared by the extrusion method. Lipids in chloroform were mixed in a glass tube and dried as thin films under a stream of nitrogen gas. 1-2 mol \% of 5-SASL was added to the lipid mixture. To remove residual organic solvent, the lipid films were further dried using a vacuum pump for $\sim 16 \mathrm{~h}$. The lipids were resuspended in a HEPES buffer (10 mM HEPES, $\mathrm{pH} 7.4$ ) by vortexing for $1-2 \mathrm{~min}$ and then subjected to $\geq 6$ freeze-and-thaw cycles. The lipid suspension was then extruded $\geq 30$ times through a mini extruder with a $100 \mathrm{~nm}$ polycarbonate membrane (Avanti Polar Lipids).

5.2.2. Lipid Bilayer Fluidity Measurements. Lipid bilayer fluidity measurements were carried out in X-band $(9.5 \mathrm{GHz})$ on a Bruker E680 continuous-wave and pulsed EPR spectrometer with a $4119 \mathrm{HS}$ high-sensitivity resonator (Bruker, Billerica, MA) at the National High Magnetic Field Laboratory (NHMFL). EPR spectra were acquired with 100 $\mathrm{kHz}$ modulation frequency, $2.4 \mathrm{~mW}$ incident microwave power (18 dB), $0.16 \mathrm{mT}$ modulation amplitude, $81.92 \mathrm{~ms}$ time constant, $81.92 \mathrm{~ms}$ conversion time, and $16 \mathrm{mT}$ scan width. Samples were loaded in $0.6 \mathrm{~mm}$ inner-diameter glass capillary tubes. Liposomes with $1 \mathrm{~mol} \%$ of 5-SASL were prepared in a $\mathrm{pH} 7.4$ HEPES buffer as described earlier with a total of 10 $\mathrm{mM}$ lipid concentration. The protein solution was mixed with liposomes to achieve the desired $\mathrm{P} / \mathrm{L}$ ratios.

5.2.3. Lipid Bilayer Polarity Measurements. Lipid bilayer polarity was determined by EPR power saturation experiments in X-band $(9.5 \mathrm{GHz})$ on a Bruker E680 spectrometer with a loop-gap resonator (Molecular Specialties, Milwaukee, WI) at the NHMFL. The samples were loaded in gas-permeable TPX capillary tubes (Molecular Specialties) and purged using a stream of either air or nitrogen $\left(\mathrm{N}_{2}\right)$ gas. EPR spectra were collected at room temperature with $100 \mathrm{kHz}$ modulation frequency, $0.16 \mathrm{mT}$ modulation amplitude, $10.24 \mathrm{~ms}$ time constant, $20.48 \mathrm{~ms}$ conversion time, and $0.35 \mathrm{mT}$ scan width centered on the central peak. The microwave power was ramped up automatically from 0.06 to $118 \mathrm{~mW}$, averaging the spectrum at each power for nine times. Each power saturation experiment consisted of three sets of spectra: first, the spectra were collected on the sample under a stream of air to ensure membrane contact with oxygen $\left(\mathrm{O}_{2}\right)$; second, the sample was purged for at least 20 min with a stream of $\mathrm{N}_{2}$ and then the spectra were collected; third, the sample with $50 \mathrm{mM}$ of nickel(II) ethylenediaminediacetate (NiEDDA) was purged for at least $20 \mathrm{~min}$ with a stream of $\mathrm{N}_{2}$ and then the spectra were collected. The obtained saturation curves (peak-to-peak amplitude vs microwave power) were fitted with a standard equation ${ }^{34,86}$ to derive the saturation parameters $P_{1 / 2}$ for $\mathrm{O}_{2}$, $\mathrm{N}_{2}$, and NiEDDA spectra. Based on $P_{1 / 2}$, the accessibility of spin probes to relaxing agents (i.e., $\mathrm{O}_{2}$ and NiEDDA) can be estimated by an accessibility parameter $(\Pi) .^{34}$ The dimensionless polarity (or depth) parameter $\Phi$ was calculated from the ratio of the accessibility values $\Pi\left(\mathrm{O}_{2}\right)$ to $\Pi$ (NiEDDA). ${ }^{34}$

\section{ASSOCIATED CONTENT}

\section{Supporting Information}

The Supporting Information is available free of charge on the ACS Publications website at DOI: 10.1021/acsomega. 8 b01462.

Image analysis of nanoscale particles observed in a POPC/POPG 4:1 bilayer after treatment with $\alpha$ Syn (Figure S1); image analysis of nanoparticles observed in a POPC bilayer after treatment with $\alpha$ Syn (Figure S2); image analysis of nanoparticles observed in a POPC + $30 \%$ Chol bilayer after treatment with $\alpha$ Syn (Figure S3); image analysis of nanoparticles observed in a POPC + $15 \%$ POPE bilayer after treatment with $\alpha \mathrm{Syn}$ (Figure S4); AFM images of a POPC + 40\% POPE bilayer exposed to $\alpha$ Syn (Figure S5); EPR spectra of liposomes labeled with 5-SASL (Figure S6) (PDF)

\section{AUTHOR INFORMATION}

\section{Corresponding Authors}

*E-mail: panj@usf.edu (J.P.).

*E-mail: song@magnet.fsu.edu (L.S.).

ORCID

Jianjun Pan: 0000-0002-7042-0022

Notes

The authors declare no competing financial interest.

\section{ACKNOWLEDGMENTS}

This work was partly supported by the National Institutes of Health Award Number 1R15GM117531-01 to J.P. and the NHMFL UCGP Grant Number 5080 and the National Institutes of Health Award Number 5R03AI122860 to L.S. The EPR data were collected at the NHMFL supported by the NSF DMR-1157490 and the State of Florida.

\section{REFERENCES}

(1) Moore, D. J.; West, A. B.; Dawson, V. L.; Dawson, T. M. Molecular pathophysiology of Parkinson's disease. Annu. Rev. Neurosci. 2005, 28, 57-87.

(2) Thomas, B.; Beal, M. F. Parkinson's disease. Hum. Mol. Genet. 2007, 16, R183-R194.

(3) Spillantini, M. G.; Schmidt, M. L.; Lee, V. M. Y.; Trojanowski, J. Q.; Jakes, R.; Goedert, M. $\alpha$-synuclein in Lewy bodies. Nature 1997, 388, 839-840.

(4) Spillantini, M. G.; Crowther, R. A.; Jakes, R.; Hasegawa, M.; Goedert, M. $\alpha$-synuclein in filamentous inclusions of Lewy bodies from Parkinson's disease and dementia with Lewy bodies. Proc. Natl. Acad. Sci. U.S.A. 1998, 95, 6469-6473.

(5) Polymeropoulos, M. H.; Lavedan, C.; Leroy, E.; Ide, S. E.; Dehejia, A.; Dutra, A.; Pike, B.; Root, H.; Rubenstein, J.; Boyer, R.; Stenroos, E. S.; Chandrasekharappa, S.; Athanassiadou, A.; Papapetropoulos, T.; Johnson, W. G.; Lazzarini, A. M.; Duvoisin, R. C.; DiIorio, G.; Golbe, L. I.; Nussbaum, R. L. Mutation in the $\alpha$ synuclein gene identified in families with Parkinson's disease. Science 1997, 276, 2045-2047.

(6) Ibáñez, P.; Bonnet, A. M.; Debarges, B.; Lohmann, E.; Tison, F.; Agid, Y.; Durr, A.; Brice, A.; Pollak, P. Causal relation between $\alpha$ synuclein gene duplication and familial Parkinson's disease. Lancet 2004, 364, 1169-1171.

(7) Singleton, A. B.; Farrer, M.; Johnson, J.; Singleton, A.; Hague, S.; Kachergus, J.; Hulihan, M.; Peuralinna, T.; Dutra, A.; Nussbaum, R.; Lincoln, S.; Crawley, A.; Hanson, M.; Maraganore, D.; Adler, C.; Cookson, M. R.; Muenter, M.; Baptista, M.; Miller, D.; Blancato, J.; Hardy, J.; Gwinn-Hardy, K. $\alpha$-synuclein locus triplication causes Parkinson's disease. Science 2003, 302, No. 841. 
(8) Davidson, W. S.; Jonas, A.; Clayton, D. F.; George, J. M. Stabilization of $\alpha$-synuclein secondary structure upon binding to synthetic membranes. J. Biol. Chem. 1998, 273, 9443-9449.

(9) Varkey, J.; Mizuno, N.; Hegde, B. G.; Cheng, N. Q.; Steven, A. C.; Langen, R. $\alpha$-Synuclein Oligomers with Broken Helical Conformation Form Lipoprotein Nanoparticles. J. Biol. Chem. 2013, 288, 17620-17630.

(10) Ulmer, T. S.; Bax, A.; Cole, N. B.; Nussbaum, R. L. Structure and dynamics of micelle-bound human $\alpha$-synuclein. J. Biol. Chem. 2005, 280, 9595-9603.

(11) Jao, C. C.; Hegde, B. G.; Chen, J.; Haworth, I. S.; Langen, R. Structure of membrane-bound $\alpha$-synuclein from site-directed spin labeling and computational refinement. Proc. Natl. Acad. Sci. U.S.A. 2008, 105, 19666-19671.

(12) Georgieva, E. R.; Ramlall, T. F.; Borbat, P. P.; Freed, J. H.; Eliezer, D. Membrane-bound $\alpha$-synuclein forms an extended helix: Long-distance pulsed ESR measurements using vesicles, bicelles, and rodlike micelles. J. Am. Chem. Soc. 2008, 130, 12856-12857.

(13) Pfefferkorn, C. M.; Jiang, Z. P.; Lee, J. C. Biophysics of $\alpha$ synuclein membrane interactions. Biochim. Biophys. Acta, Biomembr. 2012, 1818, 162-171.

(14) Kamp, F.; Exner, N.; Lutz, A. K.; Wender, N.; Hegermann, J.; Brunner, B.; Nuscher, B.; Bartels, T.; Giese, A.; Beyer, K.; Eimer, S.; Winklhofer, K. F.; Haass, C. Inhibition of mitochondrial fusion by $\alpha$ synuclein is rescued by PINK1, Parkin and DJ-1. EMBO J. 2010, 29, 3571-3589.

(15) Gosavi, N.; Lee, H. J.; Lee, J. S.; Patel, S.; Lee, S. J. Golgi fragmentation occurs in the cells with prefibrillar $\alpha$-synuclein aggregates and precedes the formation of fibrillar inclusion. J. Biol. Chem. 2002, 277, 48984-48992.

(16) Smith, W. W.; Jiang, H. B.; Pei, Z.; Tanaka, Y.; Morita, H.; Sawa, A.; Dawson, V. L.; Dawson, T. M.; Ross, C. A. Endoplasmic reticulum stress and mitochondrial cell death pathways mediate A53T mutant $\alpha$-synuclein-induced toxicity. Hum. Mol. Genet. 2005, 14, $3801-3811$.

(17) Haass, C.; Selkoe, D. J. Soluble protein oligomers in neurodegeneration: lessons from the Alzheimer's amyloid betapeptide. Nat. Rev. Mol. Cell Biol. 2007, 8, 101-112.

(18) Arrasate, M.; Mitra, S.; Schweitzer, E. S.; Segal, M. R.; Finkbeiner, S. Inclusion body formation reduces levels of mutant huntingtin and the risk of neuronal death. Nature 2004, 431, 805810.

(19) Kramer, M. L.; Schulz-Schaeffer, W. J. Presynaptic $\alpha$-synuclein aggregates, not Lewy bodies, cause neurodegeneration in dementia with Lewy bodies. J. Neurosci. 2007, 27, 1405-1410.

(20) Conway, K. A.; Lee, S. J.; Rochet, J. C.; Ding, T. T.; Williamson, R. E.; Lansbury, P. T. Acceleration of oligomerization, not fibrillization, is a shared property of both $\alpha$-synuclein mutations linked to early-onset Parkinson's disease: Implications for pathogenesis and therapy. Proc. Natl. Acad. Sci. U.S.A. 2000, 97, 571-576. (21) Vekrellis, K.; Xilouri, M.; Emmanouilidou, E.; Rideout, H. J.; Stefanis, L. Pathological roles of $\alpha$-synuclein in neurological disorders. Lancet Neurol. 2011, 10, 1015-1025.

(22) West, A.; Brummel, B. E.; Braun, A. R.; Rhoades, E.; Sachs, J. $\mathrm{N}$. Membrane remodeling and mechanics: Experiments and simulations of $\alpha$-Synuclein. Biochim. Biophys. Acta, Biomembr. 2016, 1858, 1594-1609.

(23) Varkey, J.; Isas, J. M.; Mizuno, N.; Jensen, M. B.; Bhatia, V. K.; Jao, C. C.; Petrlova, J.; Voss, J. C.; Stamou, D. G.; Steven, A. C.; Langen, R. Membrane Curvature Induction and Tubulation Are Common Features of Synucleins and Apolipoproteins. J. Biol. Chem. 2010, 285, 32486-32493.

(24) Westphal, C. H.; Chandra, S. S. Monomeric Synucleins Generate Membrane Curvature. J. Biol. Chem. 2013, 288, 1829-1840.

(25) Jiang, Z.; de Messieres, M.; Lee, J. C. Membrane Remodeling by $\alpha$-Synuclein and Effects on Amyloid Formation. J. Am. Chem. Soc. 2013, 135, 15970-15973.

(26) Mizuno, N.; Varkey, J.; Kegulian, N. C.; Hegde, B. G.; Cheng, N. Q.; Langen, R.; Steven, A. C. Remodeling of Lipid Vesicles into
Cylindrical Micelles by $\alpha$-Synuclein in an Extended $\alpha$-Helical Conformation. J. Biol. Chem. 2012, 287, 29301-29311.

(27) Rohrbough, J.; Broadie, K. Lipid regulation of the synaptic vesicle cycle. Nat. Rev. Neurosci. 2005, 6, 139-150.

(28) Kučerka, N.; Nieh, M. P.; Katsaras, J. Fluid phase lipid areas and bilayer thicknesses of commonly used phosphatidylcholines as a function of temperature. Biochim. Biophys. Acta, Biomembr. 2011, 1808, 2761-2771.

(29) Kučerka, N.; Holland, B. W.; Gray, C. G.; Tomberli, B.; Katsaras, J. Scattering Density Profile Model of POPG Bilayers As Determined by Molecular Dynamics Simulations and Small-Angle Neutron and X-ray Scattering Experiments. J. Phys. Chem. B 2012, $116,232-239$.

(30) Hubbell, W. L.; McConnell, H. M. Orientation and motion of amphiphilic spin labels in membranes. Proc. Natl. Acad. Sci. U. S. A. 1969, 64, 20-27.

(31) Hubbell, W. L.; McConnell, H. M. Molecular motion in spinlabeled phospholipids and membranes. J. Am. Chem. Soc. 1971, 93, 314-326.

(32) Bortolus, M.; Parisio, G.; Maniero, A. L.; Ferrarini, A. Monomeric Fullerenes in Lipid Membranes: Effects of Molecular Shape and Polarity. Langmuir 2011, 27, 12560-12568.

(33) Bortolus, M.; Dalzini, A.; Toniolo, C.; Hahm, K. S.; Maniero, A. L. Interaction of hydrophobic and amphipathic antimicrobial peptides with lipid bicelles. J. Pept. Sci. 2014, 20, 517-525.

(34) Altenbach, C.; Greenhalgh, D. A.; Khorana, G. H.; Hubbell, W. L. A collision gradient method to determine the immersion depth of nitroxides in lipid bilayers: Application to spin-labeled mutants of bacteriorhodopsin. Proc. Natl. Acad. Sci. U.S.A. 1994, 91, 1667-1671.

(35) Galvagnion, C.; Brown, J. W. P.; Ouberai, M. M.; Flagmeier, P.; Vendruscolo, M.; Buell, A. K.; Sparr, E.; Dobson, C. M. Chemical properties of lipids strongly affect the kinetics of the membraneinduced aggregation of $\alpha$-synuclein. Proc. Natl. Acad. Sci. U.S.A. 2016, 113, 7065-7070.

(36) Jo, E.; McLaurin, J.; Yip, C. M.; George-Hyslop, P.; Fraser, P. E. $\alpha$-synuclein membrane interactions and lipid specificity. J. Biol. Chem. 2000, 275, 34328-34334.

(37) Zhu, M.; Li, J.; Fink, A. L. The association of $\alpha$-synuclein with membranes affects bilayer structure, stability, and fibril formation. J. Biol. Chem. 2003, 278, 40186-40197.

(38) Pan, J.; Sahoo, P. K.; Dalzini, A.; Hayati, Z.; Aryal, C. M.; Teng, P.; Cai, J. F.; Gutierrez, H. R.; Song, L. K. Membrane Disruption Mechanism of a Prion Peptide (106-126) Investigated by Atomic Force Microscopy, Raman and Electron Paramagnetic Resonance Spectroscopy. J. Phys. Chem. B 2017, 121, 5058-5071.

(39) Shai, Y. Mechanism of the binding, insertion and destabilization of phospholipid bilayer membranes by $\alpha$-helical antimicrobial and cell non-selective membrane-lytic peptides. Biochim. Biophys. Acta, Biomembr. 1999, 1462, 55-70.

(40) Henderson, J. M.; Waring, A. J.; Separovic, F.; Lee, K. Y. C. Antimicrobial Peptides Share a Common Interaction Driven by Membrane Line Tension Reduction. Biophys. J. 2016, 111, 21762189.

(41) Shi, Z.; Sachs, J. N.; Rhoades, E.; Baumgart, T. Biophysics of $\alpha$ synuclein induced membrane remodelling. Phys. Chem. Chem. Phys. 2015, 17, 15561-15568.

(42) Madine, J.; Hughes, E.; Doig, A. J.; Middleton, D. A. The effects of $\alpha$-synuclein on phospholipid vesicle integrity: a study using 31P NMR and electron microscopy. Mol. Membr. Biol. 2008, 25, 518-527.

(43) Braun, A. R.; Lacy, M. M.; Ducas, V. C.; Rhoades, E.; Sachs, J. N. $\alpha$-Synuclein-Induced Membrane Remodeling Is Driven by Binding Affinity, Partition Depth, and Interleaflet Order Asymmetry. J. Am. Chem. Soc. 2014, 136, 9962-9972.

(44) Volles, M. J.; Lee, S. J.; Rochet, J. C.; Shtilerman, M. D.; Ding, T. T.; Kessler, J. C.; Lansbury, P. T. Vesicle permeabilization by protofibrillar $\alpha$-synuclein: Implications for the pathogenesis and treatment of Parkinson's disease. Biochemistry 2001, 40, 7812-7819. 
(45) van Rooijen, B. D.; Claessens, M. M. A. E.; Subramaniam, V. Lipid bilayer disruption by oligomeric $\alpha$-synuclein depends on bilayer charge and accessibility of the hydrophobic core. Biochim. Biophys. Acta, Biomembr. 2009, 1788, 1271-1278.

(46) Smith, D. P.; Tew, D. J.; Hill, A. F.; Bottomley, S. P.; Masters, C. L.; Barnham, K. J.; Cappai, R. Formation of a high affinity lipidbinding intermediate during the early aggregation phase of $\alpha$ synuclein. Biochemistry 2008, 47, 1425-1434.

(47) Rhoades, E.; Ramlall, T. F.; Webb, W. W.; Eliezer, D. Quantification of $\alpha$-synuclein binding to lipid vesicles using fluorescence correlation spectroscopy. Biophys. J. 2006, 90, 46924700.

(48) Pantusa, M.; Vad, B.; Lillelund, O.; Kjaer, L.; Otzen, D.; Bartucci, R. $\alpha$-synuclein and familial variants affect the chain order and the thermotropic phase behavior of anionic lipid vesicles. Biochim. Biophys. Acta, Proteins Proteomics 2016, 1864, 1206-1214.

(49) van Maarschalkerweerd, A.; Vetri, V.; Langkilde, A. E.; Fodera, V.; Vestergaard, B. Protein/Lipid Coaggregates are Formed During aSynuclein-Induced Disruption of Lipid Bilayers. Biomacromolecules 2014, 15, 3643-3654.

(50) Braun, A. R.; Sachs, J. N. $\alpha$-Synuclein Reduces Tension and Increases Undulations in Simulations of Small Unilamellar Vesicles. Biophys. J. 2015, 108, 1848-1851.

(51) Perrin, R. J.; Woods, W. S.; Clayton, D. F.; George, J. M. Interaction of human $\alpha$-synuclein and Parkinson's disease variants with phospholipids - Structural analysis using site-directed mutagenesis. J. Biol. Chem. 2000, 275, 34393-34398.

(52) Takamori, S.; Holt, M.; Stenius, K.; Lemke, E. A.; Gronborg, M.; Riedel, D.; Urlaub, H.; Schenck, S.; Brugger, B.; Ringler, P.; Muller, S. A.; Rammner, B.; Grater, F.; Hub, J. S.; De Groot, B. L.; Mieskes, G.; Moriyama, Y.; Klingauf, J.; Grubmuller, H.; Heuser, J.; Wieland, F.; Jahn, R. Molecular anatomy of a trafficking organelle. Cell 2006, 127, 831-846.

(53) Dason, J. S.; Smith, A. J.; Marin, L.; Charlton, M. P. Vesicular Sterols Are Essential for Synaptic Vesicle Cycling. J. Neurosci. 2010, 30, $15856-15865$.

(54) Thiele, C.; Hannah, M. J.; Fahrenholz, F.; Huttner, W. B. Cholesterol binds to synaptophysin and is required for biogenesis of synaptic vesicles. Nat. Cell Biol. 2000, 2, 42-49.

(55) Lundbaek, J. A.; Andersen, O. S.; Werge, T.; Nielsen, C. Cholesterol-induced protein sorting: An analysis of energetic feasibility. Biophys. J. 2003, 84, 2080-2089.

(56) Henriksen, J.; Rowat, A. C.; Brief, E.; Hsueh, Y. W.; Thewalt, J. L.; Zuckermann, M. J.; Ipsen, J. H. Universal behavior of membranes with sterols. Biophys. J. 2006, 90, 1639-1649.

(57) Pan, J.; Mills, T. T.; Tristram-Nagle, S.; Nagle, J. F. Cholesterol perturbs lipid bilayers nonuniversally. Phys. Rev. Lett. 2008, 100, No. 198103.

(58) Vance, J. E. Dysregulation of cholesterol balance in the brain: contribution to neurodegenerative diseases. Dis. Models Mech. 2012, 5, $746-755$.

(59) Fantini, J.; Carlus, D.; Yahi, N. The fusogenic tilted peptide (67-78) of $\alpha$-synuclein is a cholesterol binding domain. Biochim. Biophys. Acta, Biomembr. 2011, 1808, 2343-2351.

(60) Hsiao, J.-H.; Halliday, G. M.; Kim, W. S. $\alpha$-Synuclein Regulates Neuronal Cholesterol Efflux. Molecules 2017, 22, No. 1769.

(61) Eriksson, I.; Nath, S.; Bornefall, P.; Giraldo, A. M. V.; Ollinger, $\mathrm{K}$. Impact of high cholesterol in a Parkinson's disease model: Prevention of lysosomal leakage versus stimulation of $\alpha$-synuclein aggregation. Eur. J. Cell Biol. 2017, 96, 99-109.

(62) Middleton, E. R.; Rhoades, E. Effects of Curvature and Composition on $\alpha$-Synuclein Binding to Lipid Vesicles. Biophys. J. 2010, 99, 2279-2288.

(63) Nuscher, B.; Kamp, F.; Mehnert, T.; Odoy, S.; Haass, C.; Kahle, P. J.; Beyer, K. $\alpha$-synuclein has a high affinity for packing defects in a bilayer membrane - A thermodynamics study. J. Biol. Chem. 2004, 279, 21966-21975.
(64) Kamp, F.; Beyer, K. Binding of $\alpha$-synuclein affects the lipid packing in bilayers of small vesicles. J. Biol. Chem. 2006, 281, 92519259.

(65) Pan, J. J.; Tristram-Nagle, S.; Nagle, J. F. Effect of cholesterol on structural and mechanical properties of membranes depends on lipid chain saturation. Phys. Rev. E 2009, 80, No. 021931.

(66) Pfefferkorn, C. M.; Heinrich, F.; Sodt, A. J.; Maltsev, A. S.; Pastor, R. W.; Lee, J. C. Depth of $\alpha$-Synuclein in a Bilayer Determined by Fluorescence, Neutron Reflectometry, and Computation. Biophys. J. 2012, 102, 613-621.

(67) Jiang, Z.; Hess, S. K.; Heinrich, F.; Lee, J. C. Molecular Details of $\alpha$-Synuclein Membrane Association Revealed by Neutrons and Photons. J. Phys. Chem. B 2015, 119, 4812-4823.

(68) Vance, J. E. Thematic review series: Glycerolipids. Phosphatidylserine and phosphatidylethanolamine in mammalian cells: two metabolically related aminophospholipids. J. Lipid Res. 2008, 49, $1377-1387$.

(69) Kollmitzer, B.; Heftberger, P.; Rappolt, M.; Pabst, G. Monolayer spontaneous curvature of raft-forming membrane lipids. Soft Matter 2013, 9, 10877-10884.

(70) Boggs, J. M. Lipid Intermolecular Hydrogen-Bonding Influence on Structural Organization and Membrane-Function. Biochim. Biophys. Acta, Rev. Biomembr. 1987, 906, 353-404.

(71) Pink, D. A.; McNeil, S.; Quinn, B.; Zuckermann, M. J. A model of hydrogen bond formation in phosphatidylethanolamine bilayers. Biochim. Biophys. Acta, Biomembr. 1998, 1368, 289-305.

(72) Kučerka, N.; van Oosten, B.; Pan, J. J.; Heberle, F. A.; Harroun, T. A.; Katsaras, J. Molecular structures of fluid phosphatidylethanolamine bilayers obtained from simulation-to-experiment comparisons and experimental scattering density profiles. J. Phys. Chem. B 2015, $119,1947-1956$.

(73) Slater, S. J.; Ho, C.; Taddeo, F. J.; Kelly, M. B.; Stubbs, C. D. Contribution of Hydrogen-Bonding to Lipid Lipid Interactions in Membranes and the Role of Lipid Order - Effects of Cholesterol, Increased Phospholipid Unsaturation, and Ethanol. Biochemistry 1993, 32, 3714-3721.

(74) Pan, J.; Dalzini, A.; Song, L. K. Cholesteroland phosphatidylethanolamine lipids exert opposite effects on membrane modulations caused by the M2 amphipathic helix Biochim. Biophys. Acta, Biomembr. 2018, DOI: 10.1016/j.bbamem.2018.07.013.

(75) Ouberai, M. M.; Wang, J.; Swann, M. J.; Galvagnion, C.; Guilliams, T.; Dobson, C. M.; Welland, M. E. $\alpha$-Synuclein Senses Lipid Packing Defects and Induces Lateral Expansion of Lipids Leading to Membrane Remodeling. J. Biol. Chem. 2013, 288, 2088320895.

(76) Cannon, B.; Hermansson, M.; Gyorke, S.; Somerharju, P.; Virtanen, J. A.; Cheng, K. H. Regulation of calcium channel activity by lipid domain formation in planar lipid bilayers. Biophys. J. 2003, 85, 933-942.

(77) Neville, F.; Cahuzac, M.; Konovalov, O.; Ishitsuka, Y.; Lee, K. Y. C.; Kuzmenko, I.; Kale, G. M.; Gidalevitz, D. Lipid headgroup discrimination by antimicrobial peptide LL-37: Insight into mechanism of action. Biophys. J. 2006, 90, 1275-1287.

(78) Puchkov, D.; Haucke, V. Greasing the synaptic vesicle cycle by membrane lipids. Trends Cell Biol. 2013, 23, 493-503.

(79) Riekkinen, P.; Rinne, U. K.; Pelliniemi, T. T.; Sonninen, V. Interaction between Dopamine and Phospholipids - Studies of Substantia Nigra in Parkinson Disease Patients. Arch. Neurol. 1975, $32,25-27$.

(80) Wang, S.; Zhang, S. Y.; Liou, L. C.; Ren, Q.; Zhang, Z. J.; Caldwell, G. A.; Caldwell, K. A.; Witt, S. N. Phosphatidylethanolamine deficiency disrupts $\alpha$-synuclein homeostasis in yeast and worm models of Parkinson disease. Proc. Natl. Acad. Sci. U.S.A. 2014, 111, E3976-E3985.

(81) Jensen, P. H.; Nielsen, M. S.; Jakes, R.; Dotti, G.; Goedert, M. Binding of $\alpha$-synuclein to brain vesicles is abolished by familial Parkinson's disease mutation. J. Biol. Chem. 1998, 273, 26292-26294. 
(82) Pan, J.; Khadka, N. K. Kinetic Defects Induced by Melittin in Model Lipid Membranes: A Solution Atomic Force Microscopy Study. J. Phys. Chem. B 2016, 120, 4625-4634.

(83) Khadka, N. K.; Teng, P.; Cai, J. F.; Pan, J. J. Modulation of lipid membrane structural and mechanical properties by a peptidomimetic derived from reduced amide scaffold. Biochim. Biophys. Acta, Biomembr. 2017, 1859, 734-744.

(84) Reviakine, I.; Brisson, A. Formation of supported phospholipid bilayers from unilamellar vesicles investigated by atomic force microscopy. Langmuir 2000, 16, 1806-1815.

(85) Leonenko, Z. V.; Carnini, A.; Cramb, D. T. Supported planar bilayer formation by vesicle fusion: the interaction of phospholipid vesicles with surfaces and the effect of gramicidin on bilayer properties using atomic force microscopy. Biochim. Biophys. Acta, Biomembr. 2000, 1509, 131-147.

(86) Altenbach, C.; Froncisz, W.; Hemker, R.; Mchaourab, H.; Hubbell, W. L. Accessibility of nitroxide side chains: Absolute Heisenberg exchange rates from power saturation EPR. Biophys. J. 2005, 89, 2103-2112. 